\title{
Uneven acute non-alcoholic fatty change of crossant the liver after percutaneous transhepatic portal vein embolization in a patient with hilar cholangiocarcinoma - a case report
}

\author{
Chun-Yi Tsai ${ }^{*}$, Motoi Nojiri, Yukihiro Yokoyama, Tomoki Ebata, Takashi Mizuno and Masato Nagino
}

\begin{abstract}
Background: Portal vein embolization is essential for patients with biliary cancer who undergo extended hepatectomy to induce hypertrophy of the future remnant liver. Over 830 patients have undergone the portal vein embolization at our institution since 1990. Non-alcoholic fatty liver disease is an entity of hepatic disease characterized by fat deposition in hepatocytes. It has a higher prevalence among persons with morbid obesity, type 2 diabetes, and hyperlipidemia. Neither the mechanism of hepatic hypertrophy after portal vein embolization nor the pathophysiology of non-alcoholic fatty liver disease has been fully elucidated. Some researchers integrated the evident insults leading to progression of fatty liver disease into the multiple-hit hypothesis. Among these recognized insults, the change of hemodynamic status of the liver was never mentioned.
\end{abstract}

Case presentation: We present the case of a woman with perihilar cholangiocarcinoma who received endoscopic biliary drainage and presented to our institute for surgical consultation. A left trisectionectomy with caudate lobectomy and extrahepatic bile duct resection was indicated for curative treatment. To safely undergo left trisectionectomy, she underwent selective portal vein embolization of the liver, in which uneven acute fatty change subsequently developed. The undrained left medial sector of the liver with dilated biliary tracts was spared the fatty change. The patient underwent planned surgery without any major complications 6 weeks after the event and has since resumed a normal life. The discrepancies in fatty deposition in the different sectors of the liver were confirmed by pathologic interpretations.

Conclusion: This is the first report of acute fatty change of the liver after portal vein embolization. The sparing of the undrained medial sector is unique and extraordinary. The images and pathologic interpretations presented in this report may inspire further research on how the change of hepatic total inflow after portal vein embolization can be one of the insults leading to non-alcoholic fatty liver disease/ change.

Keywords: Non-alcoholic fatty liver disease, Portal vein embolization, Hilar cholangiocarcinoma, Biliary tract obstruction, Case report

\footnotetext{
* Correspondence: andreas3048@gmail.com

Division of Surgical Oncology, Department of Surgery, Nagoya University

Graduate School of Medicine, 65 Tsurumai-cho, Showa-ku, Nagoya 466-8550,

Japan
} 


\section{Background}

Non-alcoholic fatty liver disease (NAFLD) is a specific entity of disease representing fat accumulation in hepatocytes that ranges from simple steatosis to necroinflammatory steatohepatitis [1, 2]. By definition, the diagnosis must be made in patients who have no evidence of excessive alcohol consumption. It is becoming one of the most common hepatic diseases in Western countries, with higher prevalence among persons with morbid obesity or type 2 diabetes $[3,4]$. The pathogenesis of NAFLD is not fully elucidated, although several theories have been proposed. Regarding inflammatory steatohepatitis, or non-alcoholic steatohepatitis (NASH), the "two-hit hypothesis" postulates that an additional oxidative injury causes hepatocellular inflammation and fibrosis in patients with existing steatosis [5]. Many potential oxidative insults have been proposed as inducers of this process. Following the accumulation of studies, the more rational "multiple-hit hypothesis" which was composed of several parallel factors contributing to progression of fatty liver change has substituted the relatively simplified "two-hit hypothesis" [6]. Regard the two hypotheses, portal flow discrepancies have not been suggested as an underlying cause.

Patients with hilar cholangiocarcinoma usually present with obstructive jaundice at the time of diagnosis and require extended hepatectomy: hence, two procedures are required before the curative operation. The first is biliary drainage to decrease serum total bilirubin levels, and the second is portal vein embolization (PVE), which is necessary for the induction of hypertrophy of the future remnant liver [7, 8]. We have aggressively treated patients with biliary tract cancer and have used PVE without major complications in over 830 patients since 1990 $[9,10]$. Although the hemodynamic changes, especially the changes of portal flow after PVE were enormous, none of these patients developed acute fatty change of the liver after PVE according to our database and literature review. The following case represents an even more extraordinary picture of uneven acute fatty change of the liver after PVE, in which the left medial sector of the liver with biliary tract obstruction was spared the changes. The changes between the affected and the unaffected segments are illustrated via images and confirmed by pathologic interpretations.

\section{Case presentation}

A 55-year-old women who was neither a smoker or heavy drinker fulfilling the criteria for alcoholism was admitted to a regional hospital due to jaundice for 2 weeks. She had underlying hyperlipidemia treated only with diet control. Her body-mass index (BMI) was $26.1 \mathrm{~kg} / \mathrm{m} 2$. Laboratory tests revealed elevated liver enzymes and hyperbilirubinemia. Computed tomography (CT) demonstrated hilar obstruction with separate bile duct dilatation in the bilateral lobes of the liver. Endoscopic retrograde cholangiopancreatography (ERCP) showed an infiltrating lesion at the bile duct bifurcation, and the diagnosis of hilar cholangiocarcinoma was made after simultaneous endoscopic biopsy. Endoscopic nasobiliary drainage (ENBD) and endoscopic biliary stenting (EBS) were also performed during the same procedure (Fig. 1). She was referred to our hospital for possible surgery. Left trisectionectomy plus caudate lobectomy with extrahepatic bile duct resection was deemed necessary to achieve R0 resection. The future liver remnant (FLR) volume (volume of the right posterior sector) was $423 \mathrm{ml}(29.3 \%)$ by CT volumetry, and PVE was indicated to induce hypertrophy of the FLR.

The left and right anterior portal branches were embolized with fibrin and steel coils [9]. There were no complications after the procedure, and routine laboratory testing yielded results similar to those pre-PVE. Routine sonography of the liver was carried out on 5th day after PVE, which confirmed good portal flow in the right posterior sector and thrombosis of the embolized portal branches. The patient was readmitted 3 weeks after PVE for volumetric evaluation of the liver and an indocyanine green clearance (ICG) test. The volume of the right posterior sector had increased to $772 \mathrm{ml}(41.3 \%)$ and the ICG-K was 0.194. Unexpectedly, a repeat CT showed diffuse fatty change of the liver parenchyma, except for the left medial sector where the bile ducts were incompletely drained (Fig. 2b). Serum laboratory parameters

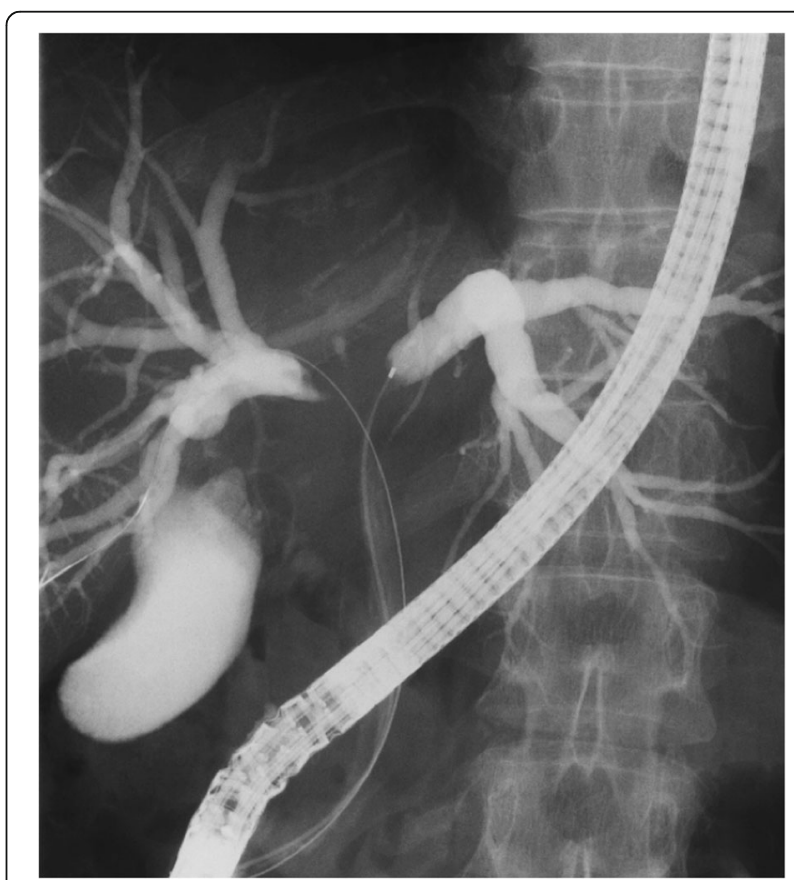

Fig. 1 Cholangiographic finding. Cholangiography after ERCP and ENBD showed that the bile ducts of segment IV of the liver were not opacified (drained) 

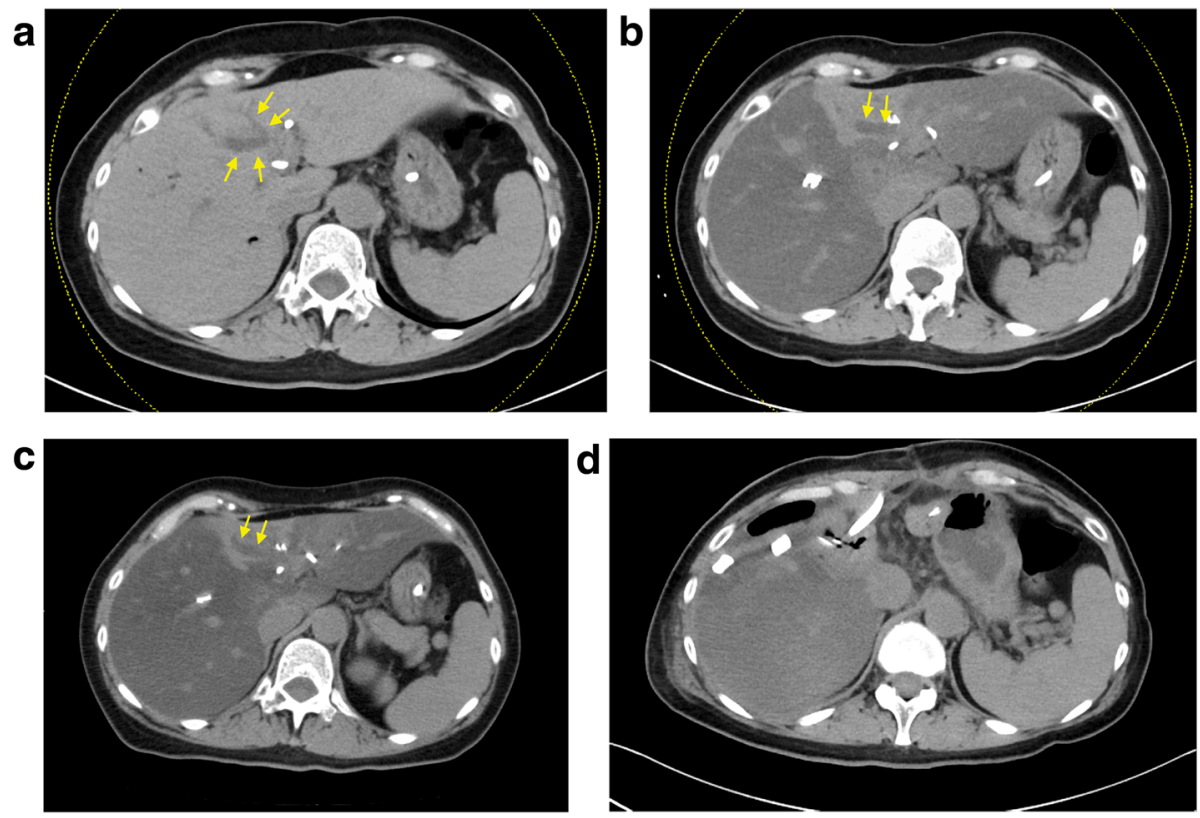

Fig. 2 CT of the liver at different stage. a CT of the liver before PVE. Yellow arrows, undrained (dilated) bile ducts (b) CT after PVE demonstrating acute fatty change of the liver and sparing of segment IV. Yellow arrows, undrained (dilated) bile ducts (c) CT 6 weeks after PVE (d) CT of the liver remnant 1 week after trisectionectomy

remained unchanged. Surgery was postponed for 3 weeks. The repeat CT still demonstrated uneven fat deposition in the liver (Fig. 2c).

Due to concern regarding cholangiocarcinoma progression, surgery was performed according to preoperative planning. Gross inspection on laparotomy revealed fatty change of the liver. The pathologic interpretation of the specimen showed different proportions of macrovesicular and microvesicular fat deposition, whereas the left medial sector showed only minimal changes (Fig. 3). The patient did not suffer hepatic dysfunction after surgery. A biliary fistula developed at the transection surface. She was discharged after resolution of the biliary fistula on postoperative day 62 and has since resumed a normal life. The course of treatment was summarized in the Table 1.

\section{Discussion}

There were two unusual observations in this case. First, to our knowledge, this is the first case of acute fatty change of the liver after PVE. Second, only the left medial sector of the liver, in which the biliary tracts were incompletely drained, was spared the fatty change, as demonstrated by both imaging and pathologic interpretation of the specimens. Although the mechanisms underlying these observations are unknown, they may have provided a connection between the change of hepatic inflow and the mechanism leading to progression of NAFLD.

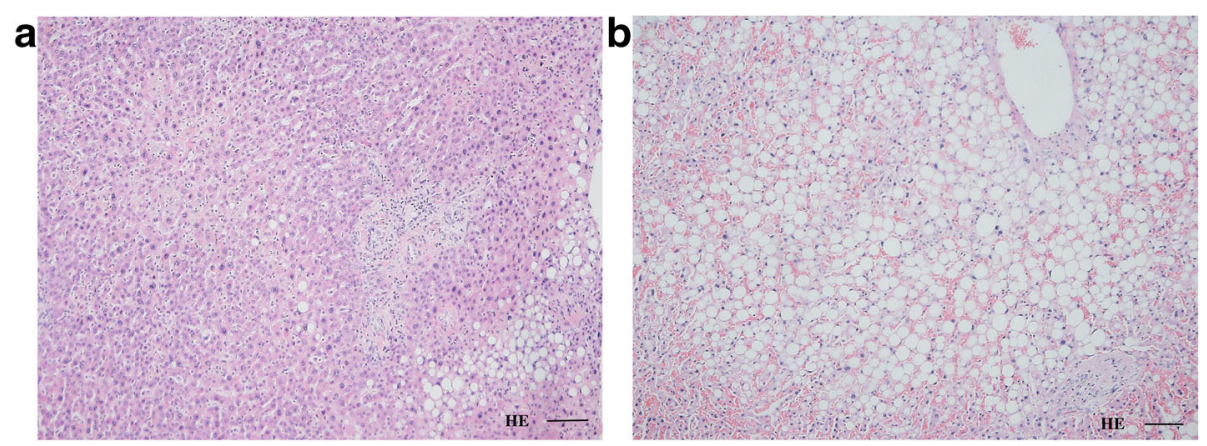

Fig. 3 Histologic aspect of different sectors of the liver. Microscopic pictures (100x, H\&E stain, scale bar =100 $\mu$ m) of fatty change in the medial sector (a) and lateral sector (b). Fat deposition is remarkable in the lateral sector 
Table 1 Timeline

\begin{tabular}{ll}
\hline July 2015 & Jaundice for 2 weeks \\
\hline August 2015 & MDCT, ERCP and biopsy confirmed hilar cholangiocarcinoma \\
& EBS and ENBD for biliary drainage \\
August 2015 & PVE for left trisectionectomy \\
September 2015 & Unevenly acute fatty change of the liver on MDCT \\
October 2015 & Stationary of fatty change of the liver \\
& Operation: left trisectionectomy and caudate lobectomy with extrahepatic bile duct resection \\
November 2015 & Surgical complication: biliary fistula \\
February 2016 & Discharged from hospital \\
\hline
\end{tabular}

$M D C T$ multidetector computed tomography, ERCP endoscopic retrograde cholangiopancreaticography, $E B S$ endoscopic biliary stent, ENBD endoscopic nasobiliary drainage, PVE portal vein embolization

NAFLD represents a broad spectrum of disease due to fat deposition in the liver, ranging from asymptomatic hepatic steatosis to necroinflammatory steatohepatitis leading to irreversible cirrhosis [11]. The manifestation of triglyceride accumulation may be due to excessive importation from the blood stream or diminished exportation and oxidation [12]. Steatosis could be detected on images; however, the diagnosis requires the specific histopathologic features demonstrated by biopsy of the liver. To delineate the mechanism of progression of NAFLD to NASH, the "multiple-hit hypothesis" that multiple parallel insults attack on genetic predisposing individuals was proposed to substitute the outdated "two-hit hypothesis" [13]. Regard the patient presented with extraordinary acute fatty deposition of the liver after PVE, which was confirmed by pathologic interpretations, we attempted to integrate the hemodynamic changes in her liver after PVE as one of the possible insults.

Based on a review of her disease course, PVE was the only major event before the uneven fatty changes of the liver. Since 1990, we have performed over 830 PVEs for patients with biliary tract cancer. This is the first patient to develop acute fatty change of the liver after PVE, either from our series or from the literature. PVE induces hemodynamic changes in both arterial and portal blood flow in both embolized and non-embolized hepatic tissue [14]. The volume of her right posterior sector increased from $423 \mathrm{ml}(29.4 \%)$ to $772 \mathrm{ml}$ (41.3\%) after PVE. The volume of the entire liver increased from $1441 \mathrm{ml}$ to $1869 \mathrm{ml}$ after PVE. The effect of PVE was remarkable in this patient.

Alterations in portal and arterial flow secondary to acute biliary tract obstruction have been observed in dogs [15]. The phenomenon is discussed mostly in patients with obstructive jaundice who receive PVE because of its negative impact on FLR hypertrophy [14]. Based on this patient's CT results and the pathologic interpretations of the specimens, it was confirmed that the incompletely drained medial sector was spared or minimally affected by the acute fatty change after PVE. It was speculated that portal venous flow was substantially decreased because of the dilated bile duct in the Glisson's sheath before PVE. Therefore, total blood flow

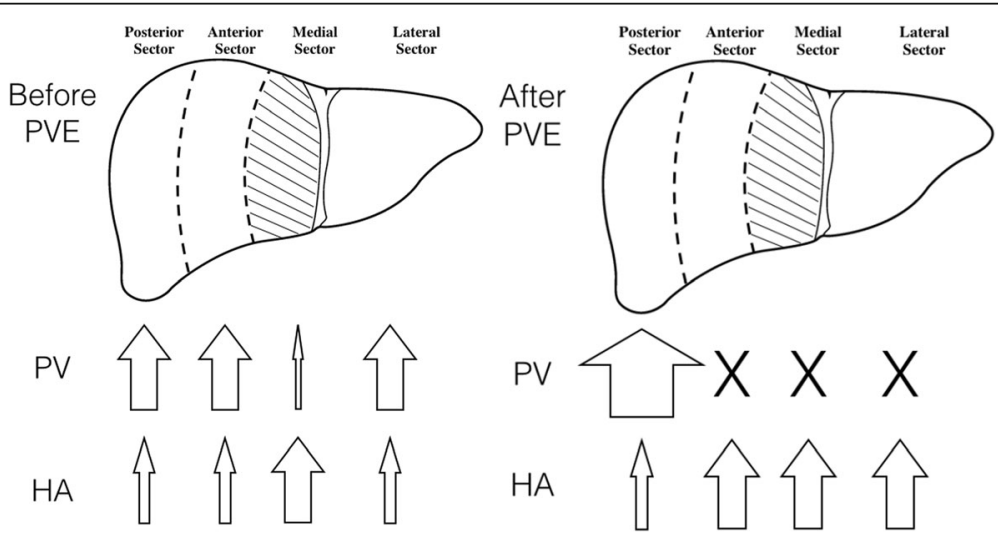

Fig. 4 Inflow of the liver before and after PVE. Illustration of the changes in arterial and the portal flow in response to PVE in the patient. PVE, portal vein embolization; PV, portal venous flow; HA, hepatic arterial flow 
in the left medial sector did not change much after PVE, which is in contrast to the dramatic blood flow changes experienced by the other sectors. In the non-embolized right posterior sector (the FLR), portal venous flow increased to accommodate blood flow from the remainder of the gastrointestinal tract after PVE. In the embolized sectors, portal venous flow was shut down, and hepatic arterial flow was increased. Hepatic arterial flow accounts for $25 \%$ of total hepatic inflow in humans and increases correspondingly to maintain constant hepatic inflow when portal flow is altered, which is known as the hepatic arterial buffer response [16]. Figure 4 illustrates the indicative, non-quantified changes in both hepatic arterial and portal flow before and after PVE among different sectors of the liver. In summary, the left medial sector experienced the least change in total inflow stemming from dilated biliary trees (unrelieved obstruction).

\section{Conclusion}

In conclusion, the uneven fatty change of the liver in this patient was extraordinary and has never been reported. We surmised that differences in hepatic inflow led to uneven sparing with respect to fatty change of the liver. Although the mechanisms underlying NAFLD and hepatic hypertrophy after PVE involve more than mere hemodynamic changes $[5,14]$, this specific presentation may give a clue for further research on the hemodynamic change of the hepatic inflow as a potential insult leading to progression of NAFLD.

\section{Abbreviations}

CT: computed tomography; EBS: endoscopic biliary stenting; ENBD: endoscopic nasobiliary drainage; ERCP: endoscopic retrograde cholangiopancreatography; FLR: future liver remnant; ICG: indocyanine green; NAFLD: non-alcoholic fatty liver disease; NASH: non-alcoholic steatohepatitis; PVE: portal vein embolization

\section{Acknowledgements}

We acknowledge all the medical staffs that took care of the patient. We also acknowledge all the surgical staffs that helped the perioperative management.

\section{Funding}

There is no funding support for this manuscript.

\section{Availability of data and materials}

All the critical images are presented in the manuscript.

\footnotetext{
Author's contributions

TE and TM designed the study and the concept. $C T$ and M Nojiri performed acquisition of data. M Nojiri and $Y Y$ analyzed the data with interpretation. CT and YY drafted the manuscript. M Nagino provided critical revision. All authors read and approved the final manuscript.
}

\section{Consent for publication}

The patient gave written consent to publish this case report and individual details.

\section{Competing interests}

There are no competing interest for any of the authors.

\section{Publisher's Note}

Springer Nature remains neutral with regard to jurisdictional claims in published maps and institutional affiliations.

Received: 24 July 2016 Accepted: 27 November 2017

Published online: 06 December 2017

\section{References}

1. Petta S, Muratore C, Craxi A. Non-alcoholic fatty liver disease pathogenesis: the present and the future. Dig Liver Dis. 2009:41(9):615-25.

2. Chalasani N, Younossi Z, Lavine JE, Diehl AM, Brunt EM, Cusi K, Charlton M, Sanyal AJ, American Gastroenterological A, American Association for the Study of liver $\mathrm{D}$, et al. The diagnosis and management of non-alcoholic fatty liver disease: practice guideline by the American Gastroenterological Association, American Association for the Study of Liver Diseases, and American College of Gastroenterology. Gastroenterology. 2012;142(7):1592-609.

3. Marchesini G, Brizi M, Morselli-Labate AM, Bianchi G, Bugianesi E, McCullough AJ, Forlani G, Melchionda N. Association of nonalcoholic fatty liver disease with insulin resistance. Am J Med. 1999;107(5):450-5.

4. Targher G, Bertolini L, Padovani R, Rodella S, Tessari R, Zenari L, Day C, Arcaro $\mathrm{G}$. Prevalence of nonalcoholic fatty liver disease and its association with cardiovascular disease among type 2 diabetic patients. Diabetes Care. 2007:30(5):1212-8.

5. Dowman JK, Tomlinson JW, Newsome PN. Pathogenesis of non-alcoholic fatty liver disease. QJM. 2010;103(2):71-83.

6. Buzzetti E, Pinzani M, Tsochatzis EA. The multiple-hit pathogenesis of nonalcoholic fatty liver disease (NAFLD). Metabolism. 2016;65(8):1038-48.

7. Nagino M, Nimura Y, Kamiya J, Kondo S, Uesaka K, Kin Y, Kutsuna Y, Hayakawa N, Yamamoto H. Right or left trisegment portal vein embolization before hepatic trisegmentectomy for hilar bile duct carcinoma. Surgery. 1995;117(6):677-81.

8. Nagino M, Kamiya J, Kanai M, Uesaka K, Sano T, Yamamoto H, Hayakawa N, Nimura Y. Right trisegment portal vein embolization for biliary tract carcinoma: technique and clinical utility. Surgery. 2000;127(2):155-60.

9. Nagino M, Nimura Y, Kamiya J, Kondo S, Kanai M. Selective percutaneous transhepatic embolization of the portal vein in preparation for extensive liver resection: the ipsilateral approach. Radiology. 1996;200(2):559-63.

10. Nagino M, Kamiya J, Nishio H, Ebata T, Arai T, Nimura Y. Two hundred forty consecutive portal vein embolizations before extended hepatectomy for biliary cancer: surgical outcome and long-term follow-up. Ann Surg. 2006; 243(3):364-72

11. Angulo P. Nonalcoholic fatty liver disease. N Engl J Med. 2002;346(16): $1221-31$.

12. Donnelly KL, Smith Cl, Schwarzenberg SJ, Jessurun J, Boldt MD, Parks EJ. Sources of fatty acids stored in liver and secreted via lipoproteins in patients with nonalcoholic fatty liver disease. J Clin Invest. 2005;115(5):1343-51.

13. Tilg $H$, Moschen AR. Evolution of inflammation in nonalcoholic fatty liver disease: the multiple parallel hits hypothesis. Hepatology. 2010;52(5):1836-46.

14. Yokoyama Y, Nagino M, Nimura Y. Mechanisms of hepatic regeneration following portal vein embolization and partial hepatectomy: a review. World J Surg. 2007;31(2):367-74.

15. Kanda H, Nimura $Y$, Yasui A, Uematsu T, Kamiya S, Machiki Y, Kitagawa $Y$, Shionoya S. Hepatic blood flow after acute biliary obstruction and drainage in conscious dogs. Hepato-Gastroenterology. 1996;43(7):235-40.

16. Lautt WW. Mechanism and role of intrinsic regulation of hepatic arterial blood flow: hepatic arterial buffer response. Am J Phys. 1985;249(5 Pt 1): G549-56.

\section{Ethic approval and consent to participate}

This study was related to the approved study of hepatobiliary surgery by UMIN Clinical Trials Registry (UMIN 000014580) and approved by the Institutional Review Board of Nagoya University Graduate School of Medicine. The patient gave written consent to participate. 